\title{
A STUDY OF THE NEMATIC-ISOTROPIC PHASE TRANSITION IN LIQUID CRYSTALS BY MONTE CARLO SIMULATIONS OF LATTICE MODELS
}

\author{
Ja. M. Ilnytskyi \\ Institute for Condensed Matter Physics of the Ukrainian Acad. Sci. \\ 1 Svientsitskii Str., Lviv, UA-290011, Ukraine
}

(Received February 5, 1997)

\begin{abstract}
The lattice models of the elongated molecules interacting via the Maier-Saupe and BernePechukas potentials are investigated. The Monte Carlo simulation of such the systems is performed in the vicinity of the nematic-isotropic (NI) transition. The internal energy, heat capacity and scalar order parameter near transition are investigated. It is shown that for more elongated molecules the NI transition becomes of a stronger first order. The results are compared both with the results of other computer simulations and with the experimental data. It is shown that the behaviour of many nematics in the vicinity of the NI transition can be described by the proposed model with the elongation ratio of molecules from 3 to 5 .
\end{abstract}

Keywords: anisotropic interactions, nematic-isotropic phase transition, Monte Carlo method.

PACS number(s): 02.70.Lq, 61.30.Cz, 64.70.Md

\section{INTRODUCTION}

Many molecular fluids consisting of anisometric molecules have one or several liquid crystalline phases [1]. The simplest among these phases is the nematic one (it appears in nematogenic liquid crystals) characterized by long-range orientational and short-range translational order. In the case of thermotropic nematogens it can be observed below the isotropic liquid phase. With the increase of temperature, the nematic to isotropic (NI) transition takes place [1].

To favour orientational ordering, the intermolecular interactions in nematogens have to be sufficiently anisotropic. In general, these anisotropic interactions consist of both long-range attractive interactions and short-range repulsive ones. This complicates a theoretical description of such systems. Maier-Saupe theory [2] is the realization of the mean-field description of the NI transition considering each molecule to be placed in the mean-field of all others. Only long-range interactions are taken into account. This theory predicts the NI transition to be of the first order. However, it overestimates the order parameter at transition and its latent heat as compared to experimental data. An alternative meanfield theory with both energetic and steric effects $[3,4]$, as well as two particle [5] and four particle [6] cluster expansions were also developed to describe the NI transition. These theoretical approaches do not include any details of the intermolecular interaction and thus are not able to describe a wide variety of real nematogens. On the other side, Onsager theory for the NI transition describes the system of long thin hard rods [7], taking into account only pure steric effects of excluded volume. This theory concludes a very strong density driven first order NI transition. Landau-de Gennes phenomenological theory of the NI transition $[1,8]$ is based on the expansion of free energy in powers of order parameter and contains a few phenomenological parameters which can be fitted with experimental data. It predicts the order of transi- tion due to symmetry considerations, and estimates pretransitional behaviour of the pair correlations and other properties [8].

A breakthrough in identifying the microscopic nature of phases in liquid crystals was achieved by computer simulations which have been initiated for these systems more than 20 years ago. There are several approaches for a computer simulation concerning the NI transition. The first one is the Monte Carlo (MC) study of the lattice version of Maier-Saupe model (presently called LebwohlLasher model). Such simulations were performed by different groups [9-14]. Molecular dynamics study of this model was also performed [15]. A MC study has been done in which the restriction to lattice sites was removed, thus adding a scalar component to the anisotropic potential [16]. This approach considers the NI transition to be temperature driven. It describes the behaviour of internal energy, heat capacity and scalar order parameter (referred further as order parameter) in the vicinity of a transition point. Furthermore, it decribes pretransitional properties of pair correlations. The soft potential used in these simulations does not include the details of molecular shape which can be important at the typical densities in real nematics.

The second approach includes MC and molecular dynamics study of hard particles systems with exclusively short-range repulsive interactions. The NI transition is interpreted as density driven. The system of hard spherocylinders $[17,18]$, spheroids $[19,20]$, thin hard platelets [21] and of other shape (see [22]) were simulated. Phase diagrams of such systems were investigated and both nematic and smectic phases were found for some molecular shapes.

Other approaches simulate systems with more realistic intermolecular potentials such as those with dispersion forces [23], Berne-Pechukas [24-26] and Gay-Berne [27] potentials. Gay-Berne potential seemed to be the most realistic for the description of liquids with elongated molecules and is used in many recent simulations. 
Adjustable parameters in this potential can be fitted to describe a real intermolecular interaction in the given mesogen. The phase diagram for Gay-Berne liquid of 256 particles was investigated by means of molecular dynamics simulations in a wide range of temperatures [28]. Computer graphics were used to identify phases and visualize configurations. The system possesses an isotropic, nematic, smectic A, smectic B and a crystal phase. Phase diagram of the similar system was also investigated using molecular dynamics simulations in the NVT ensemble [29]. The isotropic liquid, nematic and smectic B phases were identified. The temperature stability of nematic phase was also investigated. These simulations suggest that the anisotropic attractive interactions play a crucial role in the formation of smectic B phase.

The parameters of Gay-Berne potential were adjusted to fit the $p$-terphenyl, and a molecular dynamics study of 256 molecules was performed in by Luckhurst and Simmonds [30]. Isotropic, nematic and smectic A phases were observed. In other study model parameters for $p$ azoxyanisole (PAA) were adjusted and a molecular dynamics simulation of 256 molecules was done [31]. The temperature dependencies of rotational and shear viscosities are in good agreement with the experimental data. More details on related subject can be found elswere [1,32-34].

We will concentrate our attention on the results obtained by computer simulations of the Lebwohl-Lasher (LL) model. This is the system of anisosymmetric molecules confined to the sites of simple cubic lattice and interacting via the simple anisotropic potential of MaierSaupe type. Reciprocal molecular spatial orientations are considered to be the only essential degrees of freedom. Due to the head-tail isotropy in most known nematics (see [1]), the dipolar interaction plays no role in nematic ordering. The first nontrivial term in the Hamiltonian corresponds to the quadrupole-type interaction [9]:

$$
H=-\epsilon \sum_{<i j>} P_{2}\left(\cos \theta_{i j}\right)
$$

where the sum includes all pairs $\langle i, j\rangle$ of the nearest neighbours, $\theta_{i j}$ is the angle between long axes of the $i$ th and the $j$-th molecule, $\epsilon$ is the maximum interaction energy and $P_{2}(x)=\frac{1}{2}\left(3 \cos ^{2}(x)-1\right)$ is the second Legendre polynomial. It was shown by Lebwohl and Lasher that the NI transition is definitely of the first order [9]. Also, estimations for transition temperature $T_{N I}$, order parameter in nematic phase at transition temperature $S_{N I}$ and latent heat were obtained. Then this model was studied in greater detail by Jansen, Vertogen and Ypma [10]. These authors stated two aims: first, to improve the accuracy of the calculations, and second, to calculate the magnetically induced birefringence and the scattering of light by orientational fluctuations in the isotropic phase. Then, the LL model was revisited by Luckhurst and Simpson [11] to achieve more accurate data for internal energy, heat capacity and latent heat at the transition, as well as transition temperature $T_{N I}$. MC data obtained by them were extensively compared with the results obtained within the cluster theories and experimental data on real nematics. A molecular dynamics study of the LL model was performed by Zannoni and Guerra [15]. Results of MC simulation of the LL model were essentially preestimated by Fabbri and Zannoni [12]. The larger lattice of molecules $\left(30^{3}\right)$ was simulated and the number of simulation runs were significantly higher than in the previous simulations. This allowed more precise estimates for transition temperature $T_{N I}$, as well as for order parameters $\left\langle P_{2}\right\rangle \equiv S,\left\langle P_{4}\right\rangle$, internal energy and heat capacity in transition region. Particular attention was paid to pair correlations $G_{2}(r)$ and $G_{4}(r)$. The difference between $T_{N I}$ and isotropic phase limiting instability temperature $T^{*}$ was obtained with reasonable accuracy, agreeing well with the experiments on real nematics.

The LL model was revisited again by Zhang, Mouritsen and Zuckermann [13] using modern numerical techniques of analyzing phase transitions. The finite-size scaling analysis proposed by Lee and Kosterlitz [35] and Ferrenberg-Swendsen reweighting technique [36] were used. Unambiguous numerical evidence is found in favour of a weak first order transition and the presence of pseudospinodal points $T^{*}$ and $T^{* *}$, which are extremely close to $T_{N I}$. The estimate for $T_{N I}$ coincides with the result from Fabbri and Zannoni [12], and the value for transition enthalpy is in satisfactory agreement with experimental data on octylcyanobiphenyl (8CB) [37]. Pseudospinodal points $T^{*}$ and $T^{* *}$ are located by analyzing the free energy curve as the function of order parameter with two minima in the transition region. In the case for the temperatures just above the transition, the correlation functions for the order parameter fluctuations are investigated by Greeff and Lee [14]. At those temperatures for which the distribution of order parameter fluctuations is Gaussian, inverse susceptibility is found to follow Landau theory behaviour, being proportional to $\left(T-T^{*}\right)$. The value for $T^{*}$ is close to the one from Fabbri and Zannoni [12].

Despite the limited reliability of the LL model, which in fact describes a rotational order-disorder transition in a crystalline solid, it plays the role of a canonical model of a system with an orientational phase transition. To bring more physics into the LL model one has to replace the simple anisotropic potential of Maier-Saupe type in (1.1) by a much more realistic one. For example, such an attempt was made by Humpries et al [23], where the MC simulation of a lattice model with anisotropic dispersion forces (being a more realistic soft potential) was performed. But in this case, the results show that the NI transition in such a model does not differ essentially from the transition in the LL model. The order parameter at transition is slightly higher and this produces a larger entropy of the transition, but both these values are insensitive to varying the relative anisotropy in the polarizability of the system. Although the authors of this paper believe these differences to be significant, they emphasized that the transition is blurred by the relatively small ensemble used in the simulations $\left(10^{3}\right.$ particles $)$. 
It is evident in the case of increasing the system's size and the use of realistic potentials the simulations become very time consuming. Most computer simulations on Gay-Berne fluids are performed on 256 particle system [28-31], being too small for investigating phase transitions. For this reason, models of the same level of simplicity as the LL model still seemed to be useful for the investigation of different phase transitions in anisotropic systems. The aim of this report is to modify the simple anisotropic potential of Maier-Saupe type used in the LL model by including the additional parameter of molecular elongation. The influence of this factor on phase transitions in hard particles systems was already investigated [22]. We will investigate the influence of this factor in the system of particles interacting via a soft potential. This will be done by using the angular part of BernePechukas potential [24] instead of a simple anisotropic potential, as used in the LL model. This potential [24] is the single-site approximation of intermolecular site-site atomic potential between linear molecules. It describes the pairwise interaction between two ellipsoids of revolution with the given elongation. The potential of MaierSaupe type can be reproduced from this potential in the limit of almost spherical molecules (we will refer to the lattice model with Berne-Pechukas potential as the modified LL (MLL) model). Comparing results of our simulations with experimental data for some nematics in the vicinity of the NI transition gives us optimism that the LL model modified in such a way yields the possibility to simulate different real systems closer to their nature.

The outline of this report is as follows. Section 2 contains the description of the simulation method on the LL model. We decided to perform our own simulations (despite considerable data available in the literature). First, test our procedure on a well known model. Second, remove any method-relative effects when comparing the simulation of the LL model with the simulation of the MLL model. Section 3 contains the definition of the MLL model and its simulation for different elongations of molecules according to the method described in section 2. The influence of molecular elongation on latent heat and order parameter is discussed. Section 4 contains comparisons of our data with the experiments on real nematics. We compare both latent heat and order parameter at the NI transition. We also extrapolate the temperature dependence of order parameter to get the effective index $\beta$. Finally, this section summarizes our general conclusions too.

\section{MONTE CARLO SIMULATION OF LEBWOHL-LASHER MODEL}

Here we present the details of Monte Carlo simulations of the LL model in the vicinity of the NI transition. We consider the system of $20^{3}$ molecules confined to the sites of simple cubic lattice with the periodic boundary conditions. Each $i$-th molecule is characterized by the unit vector $\vec{u}_{i}$ directed along its long axis. Each direc- tion $\vec{u}_{i}$ is stored as a set of its Cartesian coordinates $\left(x_{i}, y_{i}, z_{i}\right)$. Pairwise interactions between nearest neighbours are considered to be of a simple anisotropic form (1.1).

A simulations at each temperature is started from the same initial perfectly ordered state along the $O Z$ axis. Then we allow each molecule to rotate to a new direction $\vec{u}_{i}$. To satisfy the ergodicity condition we have to move in the phase space of configurations by reasonable steps. Thus, the new direction ${\overrightarrow{u^{\prime}}}_{i}$ can be chosen in the following way:

$$
{\overrightarrow{u^{\prime}}}_{i}=\left(x_{i}^{\prime}, y_{i}^{\prime}, z_{i}^{\prime}\right)=\left(\frac{x_{i}+\delta \cdot p_{1}}{l_{u^{\prime}}}, \frac{y_{i}+\delta \cdot p_{2}}{l_{u^{\prime}}}, \frac{z_{i}+\delta \cdot p_{3}}{l_{u^{\prime}}}\right)
$$

where $\delta<1$ is the restriction parameter, $l_{u^{\prime}}^{2}=\left(x_{i}+\delta\right.$. $\left.p_{1}\right)^{2}+\left(y_{i}+\delta \cdot p_{2}\right)^{2}+\left(z_{i}+\delta \cdot p_{3}\right)^{2}$ stays for the normalization of $\overrightarrow{u^{\prime}}{ }_{i}, p_{1,2,3}$ are the pseudorandom numbers from -1 to +1 , generated by [38]:

$$
\begin{gathered}
p_{n+1}^{(\mathrm{fl})}=2.0 \cdot \frac{x_{n+1}^{(\mathrm{f})}}{m^{(\mathrm{fl})}}-1.0, \\
x_{n+1}^{(\mathrm{int})}=\left(a^{(\mathrm{int})} \cdot x_{n}^{(\mathrm{int})}\right) \& m^{(\mathrm{int})} .
\end{gathered}
$$

Here $a=16807, m=2^{31}-1$, index (int) denotes 32-bit integer variable, index (f) denotes a floating-point variable, \& is bitwise $A N D$ operation between the integers. We use three independent generators of (2.4) type for each of $p_{1,2,3}$ started from a different initial $x_{0}$.

The acceptance or rejection of a new configuration is considered with the accordance of standard Metropolis algorithm [38]. The restriction parameter $\delta$ limits the maximum possible rotation of the molecule when moving from the previous to new state and controls the acceptance-rejection ratio. This is similar to the restriction for the spherical polar angle when working in the spherical frame [11]. One MC cycle is completed after allowing all molecules to change their orientation. We performed $10^{5}$ cycles for the temperatures close to transition. The dimensionless single-molecule internal energy is defined by:

$$
U^{*}=\frac{<H>}{N \cdot \epsilon}
$$

(where $H$ is defined in (1.1)). Our simulation data for $U^{*}$ is presented in fig. 1 and coincides well with others for the same system size [11]. Simple statistical averaging for calculating $U^{*}$ in (2.5) (open circles in fig. 1) produces a curve with the change of slope at inverse transition temperature $\beta_{N I} \epsilon$. To find the latent heat of the transition, we followed the common method of using histograms of 
the energy distribution [12]. In the vicinity of transition, these histograms demonstrate two-hill shapes which indicate the coexistence of isotropic and nematic phases (fig. 2). Corresponding cumulants of these distributions were discussed in detail previously [12]. We can separate energy values of two phases by estimating maximums of these histograms, which are shown in fig. 1 by triangles. Our estimation for the latent heat in the case of the LL model is $\Delta U=\Delta U^{*} \varepsilon=0.062 \varepsilon$, which is in agreement with the values $\Delta U=0.1 \varepsilon[10], \Delta U=0.07 \varepsilon[11]$ and $\Delta U=0.05 \varepsilon[12]$.

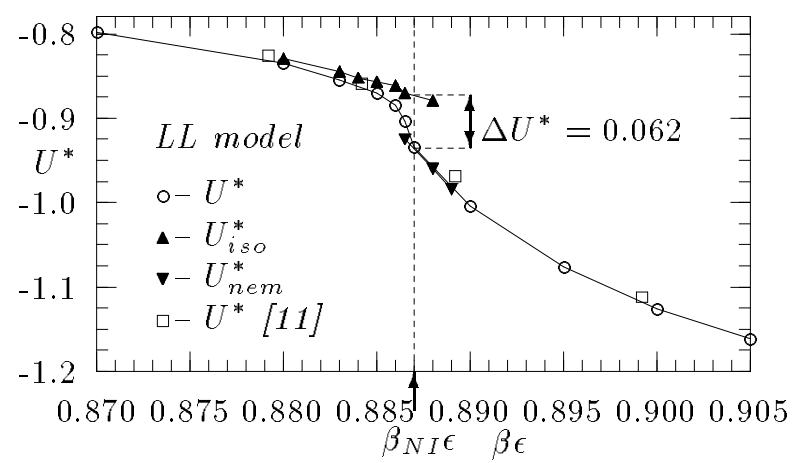

Fig. 1. Dimensionless single-molecule internal energy $U^{*}$ in the vicinity of the NI transition for the Lebwohl-Lasher model (open circles - averaged energy, triangles - separated energies of coexisting phases, open squares - data from [11], $\Delta U^{*}-$ dimensionless latent heat).

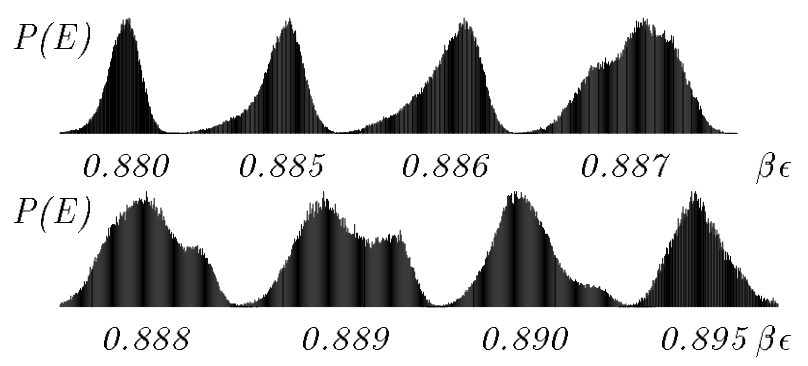

Fig. 2. Histograms for internal energy distribution in the vicinity of the NI transition for the Lebwohl-Lasher model.

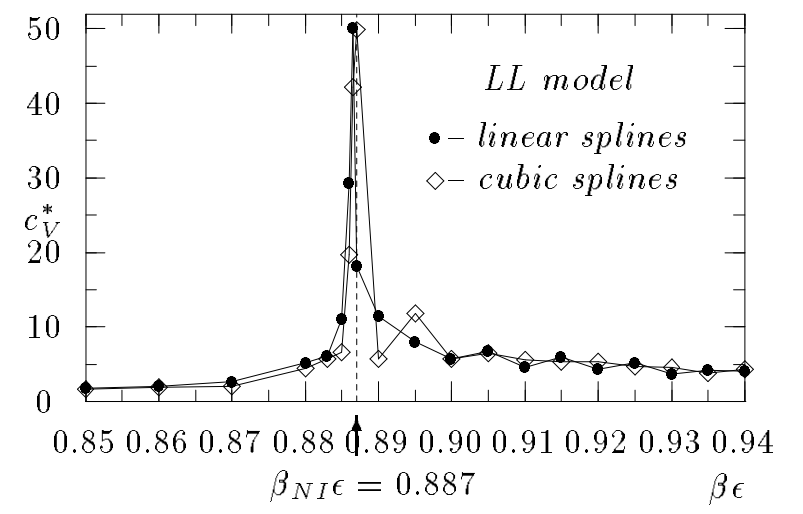

Fig. 3. Determining of the NI transition temperature from the peak heat capacity for the Lebwohl-Lasher model.
The change of slope for $U^{*}$ at the transition region leads to a peak in the single-molecule heat capacity $c_{V}^{*}$ (fig. 3):

$$
c_{V}^{*}=\frac{1}{N}\left(\frac{\partial<H>}{\partial T}\right)_{V}=-(\beta \epsilon)^{2}\left(\frac{\partial U^{*}}{\partial(\beta \epsilon)}\right)_{V} .
$$

To find this derivative, we used both differentiation of $\mathrm{cu}-$ bic splain interpolation between $U^{*}$ points with respect to $\beta \epsilon[11]$ and differentiation of linear interpolation between $U^{*}$ points. Both methods coincide well and give a peak for $c_{V}^{*}$ at $\beta_{N I} \epsilon=0.887$. This result is close enough to the previous results of $\beta_{N I} \epsilon=0.894$ [10], $\beta_{N I} \epsilon=0.887$ [11], and $\beta_{N I} \epsilon=0.890$ [12]. It should be noted that this procedure is very sensitive to the accuracy of energy data points. Therefore, the calculation of $c_{V}^{*}$ is useful primarily for estimating transition temperature and is not accurate enough to compare it with the experimental data.

One of the most important aims of MC simulation of the LL model is to evaluate a scalar order parameter [1]:

$$
S \equiv<P_{2}>=<P_{2}\left(\cos \theta_{i}\right)>
$$

where $\theta_{i}$ is the angle between the long axis of the $i$ th molecule and the director (preferred direction of nematic ordering). As it was pointed out by Fabbri and Zannoni [12], director can fluctuate from one simulation cycle to another. Thus, the order parameter is calculated with respect to the instantaneous preferred direction after a given cycle. This is normally done [10-12] by the method proposed by Viellard-Baron [17]. According to this method, the tensor order parameter [1]:

$$
S_{i j}=\frac{3}{2}\left(\begin{array}{ccc}
<x_{i}^{2}>-1 / 3 & <x_{i} y_{i}> & <x_{i} z_{i}> \\
<y_{i} x_{i}> & <y_{i}^{2}>-1 / 3 & <y_{i} z_{i}> \\
<z_{i} x_{i}> & <z_{i} y_{i}> & <z_{i}^{2}>-1 / 3
\end{array}\right)
$$

is calculated after each MC cycle and its eigenvalues $\lambda_{i}$ are found. Diagonalization of $S_{i j}$ corresponds to switching from the laboratory frame to the director frame. The largest eigenvalue of $S_{i j}$ defines the scalar order parameter

$$
S=\max \left(\lambda_{i}\right)
$$

The evaluation of $S$ during simulations shows a dramatic increase of its fluctuations in the transition region. A typical behaviour of $S$ as the function of the Monte 
Carlo cycles at $\beta \epsilon=0.886$ is shown in fig. 4 . It is evident that this figure shows the jumps between two coexisting phases, namely, isotropic and nematic, occuring simultaneously at this temperature. To separate the values for the order parameter in two coexisting phases, as before, we use histograms of the order parameter distribution. This is possible only for some temperature points with recognizable peaks. For other temperatures, the histogram maxima are not so well defined.

The behaviour of the order parameter as the function of temperature is shown in fig. 5 . We found that the order parameter in the nematic phase is $S_{N I}=0.240$ whose value is close to the previous ones of $S_{N I}=0.333$ [10], $S_{N I}=0.270$ [11], and $S_{N I}=0.270$ [12]. Most of the experimental values for $S_{N I}$ are between 0.3 and 0.45 [39]. It should be noted that the curve for $S$ in the vicinity of $\beta_{N I}$ is very steep so the value of $S_{N I}$ is very sensitive to the accuracy of $\beta_{N I}$.

Thus, we reproduced here a standard computational method for the simulation of the LL model in the vicinity of the NI transition [9-12]. That is why we will omit corresponding explanations in the next section which contain the original results. As the result, by using a unique simulational scheme, we will be able to compare simulations of the LL and the MLL models more adequately.

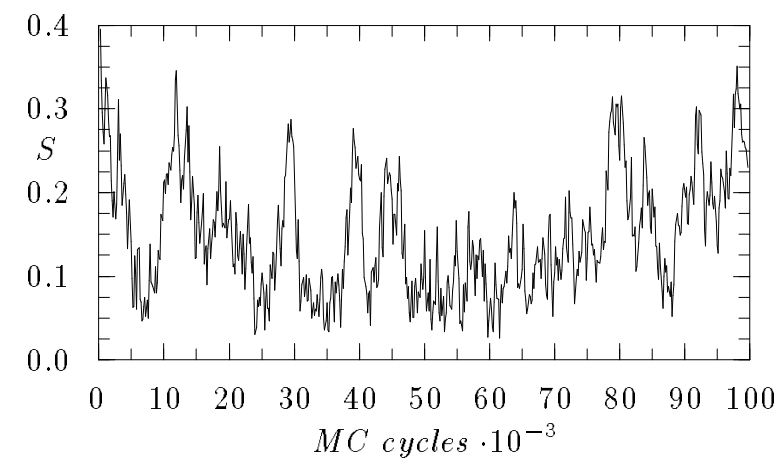

Fig. 4. Typical behaviour of the order parameter $S$ vs Monte Carlo cycles for temperatures close to the NI transition tempetature (the case of Lebwohl-Lasher model, $\beta \epsilon=0.886$ ).

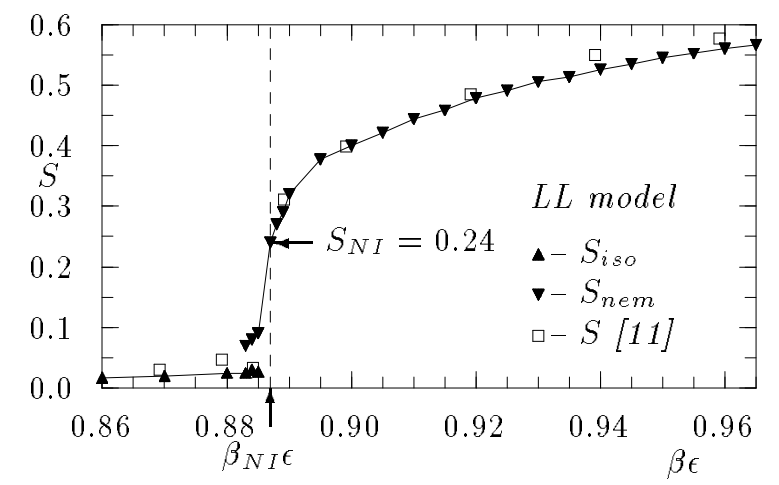

Fig. 5. Order parameter $S$ near the NI transition for the Lebwohl-Lasher model (triangles — present data, squares data from [11])

\section{MODIFIED LEBWOHL-LASHER MODEL}

As one can see, the LL model quantitative describes the NI transition in the liquid crystal materials. But the values for latent heat $\Delta U$, as well as nematic order parameter at transition point $S_{N I}$ given by the LL model, are fixed and cannot cover the entire interval of typical experimental values for real nematics [39]. In order to improve the physics of this model, one has to include additional parameters connected with the details of the intermolecular interaction. We propose that this can be done by using more realistic Berne-Pechukas (BP) potential [24]. In case we still retain the lattice, only the angular part of this potential will be used. This brings the additional parameter of molecular elongation into the model.

BP potential has the Lennard-Jones form with the orientational dependent strength and range parameters [24]:

$$
V_{B P}\left(\vec{u}_{i}, \overrightarrow{u_{j}}, \vec{r}\right)=4 \epsilon_{i j}\left[\left(\frac{\sigma_{i j}}{r}\right)^{12}-\left(\frac{\sigma_{i j}}{r}\right)^{6}\right]
$$

where

$$
\epsilon_{i j}=\epsilon_{0}\left[1-\chi^{2}\left(\overrightarrow{u_{i}} \cdot \overrightarrow{u_{j}}\right)^{2}\right]^{-1 / 2}
$$

and

$$
\sigma_{i j}=\sigma_{0}\left\{1-\frac{\chi}{2}\left[\frac{\left(\hat{r} \overrightarrow{u_{i}}+\hat{r} \overrightarrow{u_{j}}\right)^{2}}{1+\chi\left(\overrightarrow{u_{i}} \overrightarrow{u_{j}}\right)}+\frac{\left(\hat{r} \overrightarrow{u_{i}}-\hat{r} \overrightarrow{u_{j}}\right)^{2}}{1-\chi\left(\overrightarrow{u_{i}} \overrightarrow{u_{j}}\right)}\right]\right\}^{-1 / 2} .
$$

The anisotropy of molecules is characterized by the anisotropy parameter:

$$
\chi=\frac{a^{2}-1}{a^{2}+1}, \quad a=\frac{\sigma_{\|}}{\sigma_{\perp}}
$$

where $\sigma_{\|}, \sigma_{\perp}$ are major and minor axes of ellipsoids of revolution, and $a$ is the elongation parameter. The distance $r=\sigma_{i j}$ is, to good accuracy, the separation at which two molecules of relative orientation specified by unit vectors $\overrightarrow{u_{i}}, \overrightarrow{u_{j}}, \hat{r}$ touch.

Let us find BP potential in the limit of small anisotropy $\chi \ll 1$. To this end, one can perform an expansion of the square root in (3.9) in powers of $\chi$ and keep leading terms:

$$
\begin{aligned}
& \lim _{\chi \ll 1} V_{B P}\left(\vec{u}_{i}, \overrightarrow{u_{j}}, \vec{r}\right)=-\frac{3}{2}\left\{\frac { 4 } { 3 } \epsilon _ { 0 } \chi ^ { 2 } \left[\left(\frac{\sigma_{i j}}{r}\right)^{6}\right.\right. \\
& \left.\left.-\left(\frac{\sigma_{i j}}{r}\right)^{12}\right]\right\} \cos ^{2} \theta_{i j}-\left\{4 \epsilon_{0}\left[\left(\frac{\sigma_{i j}}{r}\right)^{6}-\left(\frac{\sigma_{i j}}{r}\right)^{12}\right]\right\}
\end{aligned}
$$


Let us denote the distance dependent term by $\epsilon$ :

$$
\epsilon=\frac{4}{3} \epsilon_{0} \chi^{2}\left[\left(\frac{\sigma_{i j}}{r}\right)^{6}-\left(\frac{\sigma_{i j}}{r}\right)^{12}\right]
$$

It is evident that in the case of:

$$
\begin{aligned}
& \text { 1. } \chi \ll 1, \\
& \text { 2. } \frac{\sigma_{i j}}{r}=\text { const. }
\end{aligned}
$$

this limit reproduces the simple anisotropic potential of Maier-Saupe type:

$$
\lim _{\chi \ll 1} V_{B P}\left(\overrightarrow{u_{i}}, \overrightarrow{u_{j}}, \vec{r}\right)=-\epsilon P_{2}\left(\cos \theta_{i j}\right)+\text { const. }
$$

Due to retaining the lattice, we need only the angular part of BP potential $V_{B P A}\left(\theta_{i j}\right)$. For the convinience we normalize it in such a manner that, at $\theta=0$ and $\theta=\frac{\pi}{2}$ this potential coincides with the Maier-Saupe one:

$$
V_{B P A}\left(\theta_{i j}\right)=-\frac{\epsilon}{2}\left[3 \xi\left(\frac{1}{\sqrt{1-\chi^{2} \cos ^{2} \theta_{i j}}}-1\right)-1\right]
$$

where

$$
\xi=\frac{\sqrt{1-\chi^{2}}}{1-\sqrt{1-\chi^{2}}}=2 \frac{a}{(a-1)^{2}}
$$

We will refer to lattice model with the potential (3.16) as the modified Lebwohl-Lasher (MLL) model. Within this model, one can consider more or less elongated molecules by varying the elongation parameter $a$. For larger values of $a$ the interaction potential (3.16) becomes more harder with the increasing of angle $\theta$. On the other hand, the LL model can be reproduced from the MLL model in the case of almost spherical molecules $a \rightarrow 1$ $(\chi \ll 1)$. To verify this fact numerically, we performed the simulation of the MLL model for $a=1.1$ and got exactly the same results as in the previous section.

To investigate the influence of molecular elongation on the NI transition, we performed simulations for the cases $a=3$ and $a=5$ (used also in hard particles simulations $[19,20])$. All simulations are done using the method described in the previous section. We observed a stronger first order NI transition in both cases of $a=3$ and $a=5$ as compared to the LL model. The energy in the case of $a=3$ is presented in fig. 7. To separate the energies of isotropic and nematic phases, we used histograms of energy distribution. The NI transition in the $a=5$ case demonstrates a very strong first order nature with a relatively large latent heat $\Delta U=0.474 \varepsilon$ and the absence of coexisting phases (see fig. 8). The latter fact can be explained by the large potential barrier between the two phases, which cannot be easily overcome during simula- tions.

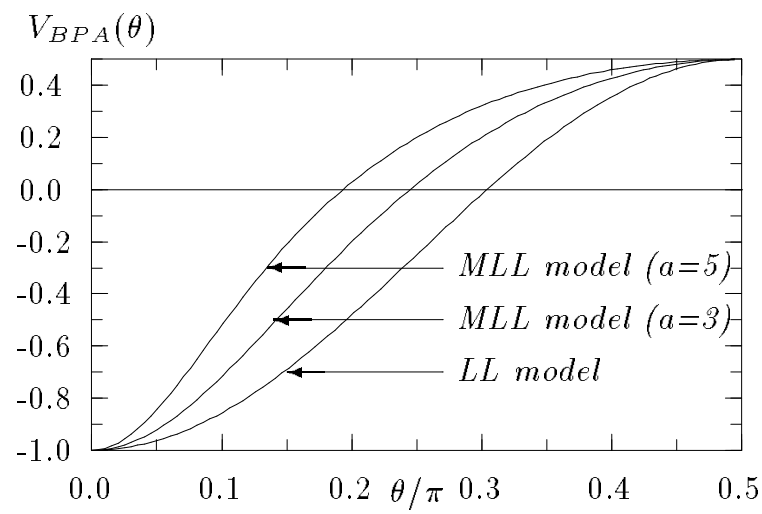

Fig. 6. Angular part of Berne-Pechukas potential $V_{B P A}(\theta)$ vs angle between molecular axes $\theta$ for different elongation of molecules (the LL model corresponds to $a \rightarrow 1$ case).

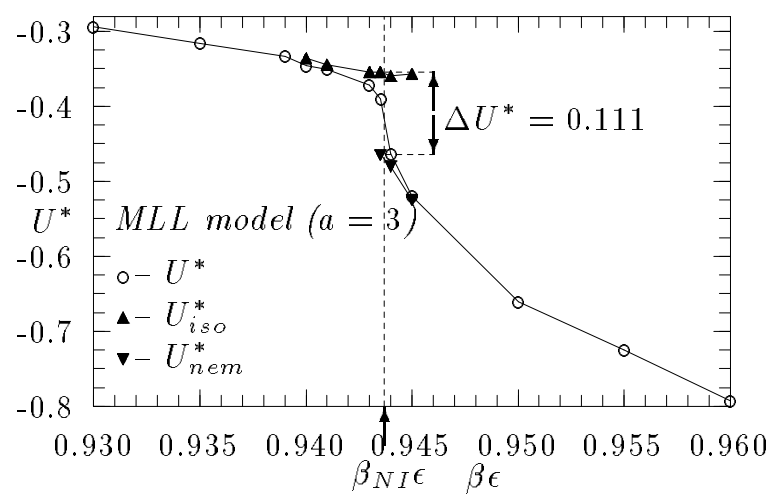

Fig. 7. Dimensionless single-molecule internal energy $U^{*}$ in the vicinity of the NI transition for the modified Lebwohl-Lasher model with the molecular elongation $a=3$ (open circles - averaged energy, triangles - separated energies of coexisting phases, $\Delta U^{*}-$ dimensionless latent heat).

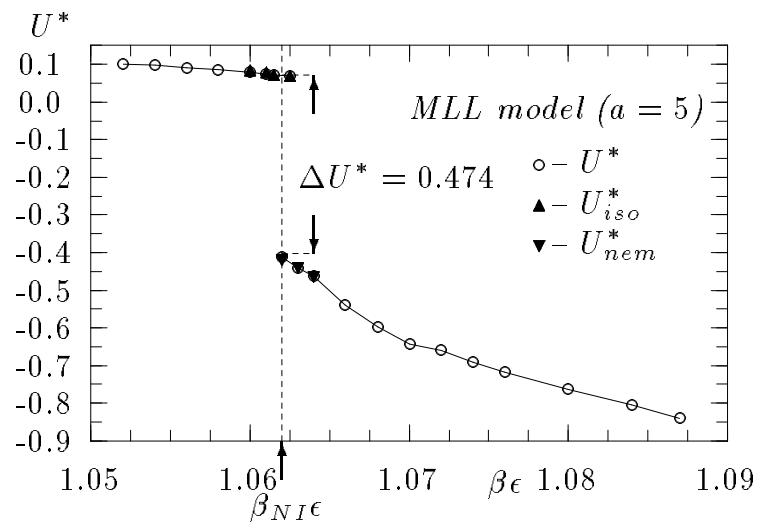

Fig. 8. Dimensionless single-molecule internal energy $U^{*}$ in the vicinity of the NI transition for the modified Lebwohl-Lasher model with the molecular elongation $a=5$ (open circles - averaged energy, triangles - separated energies of coexisting phases, $\Delta U^{*}$ - dimensionless latent heat). 


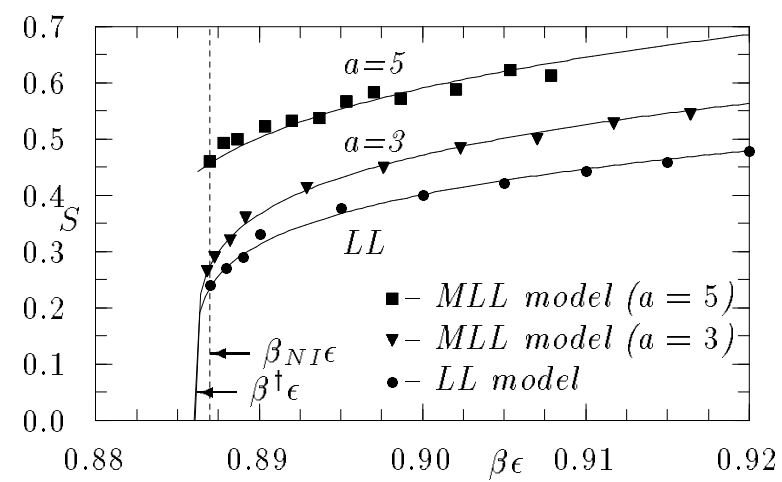

Fig. 9. Behaviour of the order parameter $S$ near the NI transition for models with different elongation of molecules ( $\beta_{N I} \epsilon-$ inverse temperature of the NI transition, $\beta^{\dagger}-$ inverse temperature of data extrapolation to fit power-law dependence).

\begin{tabular}{|l|l|l|l|}
\hline model & $\beta_{N I} \epsilon$ & $\Delta U$ & $S_{N I}$ \\
\hline LL & 0.887 & $0.062 \epsilon$ & 0.240 \\
MLL $(\mathrm{a}=3)$ & 0.9437 & $0.111 \epsilon$ & 0.285 \\
MLL $(\mathrm{a}=5)$ & 1.062 & $0.474 \epsilon$ & 0.460 \\
\hline
\end{tabular}

Table 1. Inverse transition temperature $\beta_{N I} \epsilon$, latent heat $\Delta U$, and order parameter at transition $S_{N I}$ for the NI transition in the Lebwohl-Lasher (LL) and the modified LebwohlLasher (MLL) models.

The behaviour of the order parameter $S$ in the case of the LL and the MLL models is shown in fig. 9. We obtained larger values for $S_{N I}$ with the increasing of $a$. The difference between $S_{N I}$ in the cases of $a=5$ and $a=3$ is much more essential than that in the cases of $a=3$ and the LL model. The behaviour of $S$ in the case of $a=5$ demonstrates a much stronger first order transition as compared to other cases. We collected values for the inverse transition temperature $\beta_{N I} \epsilon$, latent heat $\Delta U$, and order parameter at transition $S_{N I}$ in table 1.

Due to these results, it can be pointed out unambiguously that the NI transition in the MLL model becomes a stronger first order one with the increasing of elongation parameter $a$. It is interesting to note that computer simulations of lattice model with anisotropic dispersion forces also gave a stronger first order transition as compared to the LL model [23]. It is known [22] that as the shape becomes more elongated, we expect to see progressive strengthening of transition towards the Onsager limit [7]. These results shed new light on the comparison of MC data to the entire set of experimental data on the NI transition.

\section{COMPARISON WITH THE EXPERIMENTAL DATA}

Our data for the NI transition temperature, latent heat and the order parameter obtained within the MLL model can be compared with the experimental data on real nematics. For example, a set of experiments on 13 nematics in the vicinity of the NI transition was collected by Haller [39]. Let us discuss the behaviour of the order parameter $S$ in the vicinity of the NI transition (fig. 9). The order parameter has some non-zero value $S_{N I}$ at the NI transition temperature and rapidly goes to zero when the temperature is increased. As was pointed out in [39], one can extrapolate the gradual decrease of $S$ until it becomes zero at a hypothetical second order phase transition temperature $T^{\dagger}$. Such extrapolation yields the following relation after scaling of experimental data [39]:

$$
S=\left(1-T / T^{\dagger}\right)^{\beta}
$$

where $\beta$ is the critical index for the order parameter in a hypothetical second order transition at $T=T^{\dagger}$. In our case we can write the similar power law in terms of inverse temperature:

$$
S=S_{0}\left(\beta \epsilon-\beta^{\dagger} \epsilon\right)^{\beta}
$$

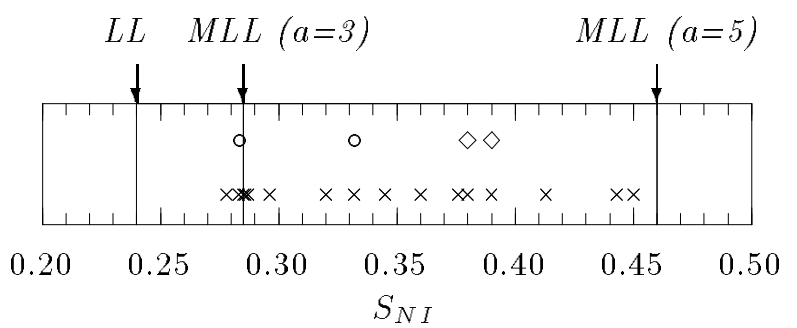

Fig. 10. The values for the order parameter at the transition $S_{N I}$ obtained within the LL and the MLL models compared to the experimental data. Circles are the particular data for MBBA, rombs - for PAA, crosses - all others (all experimental data are taken from [39]).

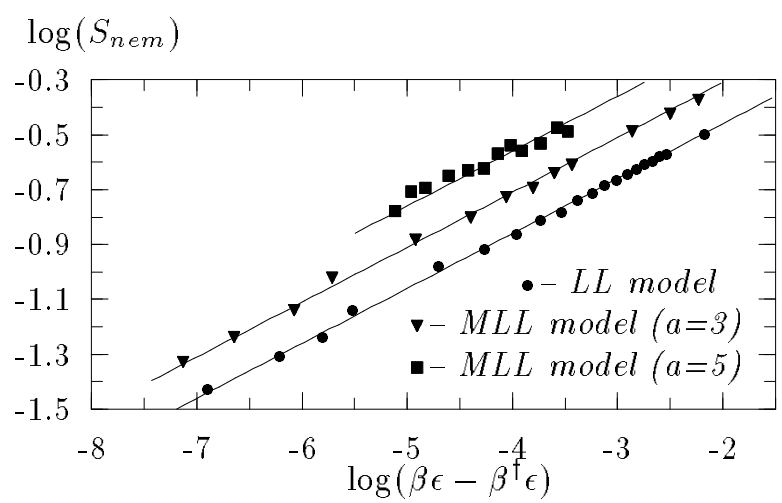

Fig. 11. Fitting data for order parameter in the nematic phase $S_{n e m}$ obtained for models with different elongation of molecules to the power law $S_{n e m}=S_{0}\left(\beta \epsilon-\beta^{\dagger} \epsilon\right)^{\beta}$ in logarithmic scale (effective index $\beta=0.2$ ).

We performed several comparisons of our results with the experimental data. First, we compared the values for $S_{N I}$ obtained for the LL and the MLL models with 
the experimental set of the respective values for $S_{N I}$. This is illustrated in fig. 10, where one can see that the typical experimental values for $S_{N I}$ turned out to be inside the interval between the values obtained for the MLL model at $a=3$ and $a=5$. Let us consider some typical nematogens. For example, from the rough steric point of view, the molecules of $p$-methoxybenzylidene- $n$ butylaniline (MBBA) correspond to the elongation $a=3$ and molecules of $p$-azoxyanisole (PAA) to $a=4$. The experimental values for MBBA $S_{N I}=0.283,0.332$ [39] are indeed close enough to our result $S_{N I}=0.285$ for $a=3$ case, and the experimental values for PAA $S_{N I}=0.38,0.39$ [39] (filled rombs in fig. 10) are exactly at the middle of the results for $a=3$ and $a=5$. One other method of comparing our results for the order parameter to the experiment lies in extrapolating our data in the nematic phase to the point $\beta^{\dagger} \epsilon$ and finding the power law (4.19). For this purpose, we plotted the dependence of $S$ as the function of $\beta \epsilon$ in the nematic phase in logarithmic scale (fig. 11). The best fits were found with the value of exponent $\beta \sim 0.2$ (solid lines in fig. 11). This value agrees well with the experimental data being in the interval of $\beta \in[0.17,0.225]$ [39].

Also comparable to the experiment is the latent heat $\Delta U$, which coincides in our case with the enthalpy of the transition $\Delta H$. We can use the experimental data obtained from studying thermal behaviour of $8 \mathrm{CB}$ by adiabatic scanning calorimeter [37]. The result for the NI transition enthalpy is $\Delta H=612 \mathrm{~J} / \mathrm{mol}$ at transition temperature $T_{N I}=40.8^{\circ} \mathrm{C}$. Fitting the transition temperature obtained for the LL and the MLL models to this value, one can get the energetic parameter $\epsilon$ and then the value for transition enthalpy. We get $\Delta H=143.23 \mathrm{~J} / \mathrm{mol}$ for the LL model, $\Delta H=272.83 \mathrm{~J} / \mathrm{mol}$ for the MLL model $(\mathrm{a}=3)$ and $\Delta H=1311.1 \mathrm{~J} / \mathrm{mol}$ for the MLL model $(\mathrm{a}=5)$. Despite the poor accuracy of this data, we can state that the experimental value for $\Delta H$ is between our results found for the MLL model at $a=3$ and $a=5$, so our results give a reasonable interval as compared to the experiment.

Thus, the modified Lebwohl-Lasher model being the lattice model of elongated molecules interacting via the soft potential of Berne-Pechukas type was proposed. The standard Metropolis Monte Carlo algorithm was used to simulate this system in the vicinity of nematic-isotropic transition. To separate the coexisting phases, the histogram technique was used. The behaviour of the internal energy, heat capacity and order parameter in the vicinity of transition was investigated. As compared to the LL model, the additional parameter of molecular elongation $a$ is present, and the influence of this parameter on the phase transition is investigated. Simulations for the cases of molecules elongation $a=3$ and $a=5$ showed unambiguously that the nematic to isotropic transition becomes a stronger first order one with the increasing of $a$. Comparing our results to the experimental data showed that many real nematics in the vicinity of nematicisotropic transition can be described well by the proposed model with the elongation of molecules from $a=3$ to $a=5$.

\section{ACKNOWLEDGEMENTS}

The author is grateful to Profs. M. Holovko, M. Kozlovskii, Drs. Yu. Holovatch and T. Krokhmalskii for valuable discussions, Drs. M. Senkus and A. Kovalenko for the help with the references and Mr. and Mrs. Washinsky for their help in preparing the text.

This work was supported in part by the State Fund for Fundamental Investigations under the program DKNT 2.4/173 of Ukrainian State Commitee for Science and Technology.
[1] P. G. de Gennes, The Physics of Liquid Crystals (Clarendon Press, Oxford, 1974).

[2] W. Maier, A. Saupe, Z. Naturforcsh. 14A, 882 (1959).

[3] B. Deloche, B. Cabane, D. Jerome, Mol. Cryst. Liq. Cryst. 15, 197 (1971).

[4] J. R. Mc Coll, C. S. Shih, Phys. Rev. Lett. 29, 85 (1972).

[5] P. Sheng, P. J. Woitowicz, Phys. Rev. A 14, 1883 (1976).

[6] R. Van der Haegen, J. Debruyne, R. Luyckx, H. N. W. Lekkerkerker, J. Chem. Phys. 73, 2469 (1980).

[7] L. Onsager, Ann. N. Y. Acad. Sci. 51, 627 (1949).

[8] E. F. Gramsbergen, L. Longa, W. H. de Jeu, Phys. Rep. 135, 195 (1986).

[9] P. A. Lebwohl, G. Lasher, Phys. Rev. A 6, 426 (1972).

[10] H. J. F. Jansen, G. Vertogen, J. G. J. Ypma, Mol. Cryst. Liq. Cryst. 38, 87 (1977).

[11] G. R. Luckhurst, P. Simpson, Mol. Phys. 47, 251 (1982).

[12] U. Fabbri, C. Zannnoni, Mol. Phys. 58, 763 (1986).

[13] Zh. Zhang, O. G. Mouritsen, M. J. Zuckermann, Phys. Rev. Lett. 69, 2803 (1992).

[14] C. W. Greeff, M. A. Lee, Phys. Rev. E 49, 3225 (1994).
[15] C. Zannoni, M. Guerra, Mol. Phys. 44, 849 (1981).

[16] G. R. Luckhurst, S. Romano, Proc. R. Soc. A 373, 111 (1980).

[17] J. Vieillard-Baron, Mol. Phys. 28, 809 (1974).

[18] D. Frenkel, J. Phys. Chem. 92, 3280 (1988).

[19] D. Frenkel, B. M. Mulder, Mol. Phys. 55, 1171 (1985).

[20] M. P. Allen, M. R. Wilson, J. Comput. Aided Mol. Des. 3, 335 (1989).

[21] R. Eppenga, D. Frenkel, Mol. Phys. 52, 1303 (1984).

[22] M. P. Allen, Phil. Trans. R. Soc. London A 344, 323 (1993).

[23] R. L. Humpries, G. R. Luckhurst and S. Romano, Mol. Phys. 42, 1205 (1981).

[24] B. J. Berne, P. Pechukas, J. Chem. Phys. 56, 4213 (1972).

[25] J. Kushick, B. J. Berne, J. Chem. Phys. 64, 1362 (1976).

[26] A. L. Tsykalo, A. D. Bagmet, Mol. Cryst. Liq. Cryst. 46, 111 (1978).

[27] J. Gay, B. J. Berne, J. Chem. Phys. 69, 3316 (1981).

[28] G. R. Luckhurst, R. A. Stephens, R. W. Phippen, Liq. Cryst. 8, 451 (1990). 
[29] E. D. Miguel, L. F. Rull, M. K. Chalam, K. E. Gubbins, Mol. Phys. 74, 405 (1991).

[30] G. R. Luckhurst, P. S. J. Simmonds, Mol. Phys. 80, 233 (1993).

[31] A. M. Smondyrev, G. B. Loriot, R. A. Pelcovits, Phys. Rev. Lett. 75, 2340 (1995).

[32] The Molecular Physics of Liquid Crystals, ed. G. R. Luckhurst and G. W. Gray (Academic Press, 1979).

[33] M. P. Allen and D. J. Tildesley, Computer Simulation of Liquids (Oxford, 1987).
[34] Phase Transitions in Liquid Crystals, ed. S. Martellucci and A. Chester (Plenum, New-York, 1992).

[35] J. Lee, J. M. Kosterlitz, Phys. Rev. Lett. 65, 137 (1990).

[36] A. M. Ferrenberg, R. H. Swendsen, Phys. Rev. Lett. 61, 2635 (1988).

[37] J. Thoen, H. Marynissen, and W. Van Dael, Phys. Rev. A 26, 2886 (1982).

[38] D. W. Heerman, Computer Simulations Methods in Theoretical Physics (Springer-Verlag, 1986).

[39] I. Haller, Progr. Sol. St. Chem. 10, 103 (1975).

\title{
ДОСЛІДЖЕННЯ ФАЗОВОГО ПЕРЕХОДУ НЕМАТИК-ІЗОТРОПНА РІДИНА В РІДКИХ КРИСТАЛАХ ШЛЯХОМ КОМП'ЮТЕРНОГО ЕКСПЕРИМЕНТУ НА ІРАТКОВИХ МОДЕЛЯХ
}

\author{
Я. М. Ільницький \\ Інститут фізики конденсованих систем НАН України \\ Україна, UA-290011, Лъвів, вул. Свениічвкого, 1
}

\begin{abstract}
Досліджено граткові моделі із видовжених молекул, які взаємодіють між собою за допомогою потенціялів типу Майера-Заупе та Берне-Печукаса. За допомогою моделювання методом Монте Карло досліджено поведінку внутрішньої енергії, теплоємності та скалярного параметру впорядкування в околі переходу нематик-ізотропна (НI) система. Показано, що при зростанні видовження молекул НІ перехід стає сильнішим переходом першого роду. Результати порівнюються із іншими даними комп'ютерного моделювання та з експериментом. Показано, що поведінка багатьох нематиків в околі НІ переходу може бути описана запропонованою моделлю із відносним видовженням молекул від 3 до 5.
\end{abstract}

